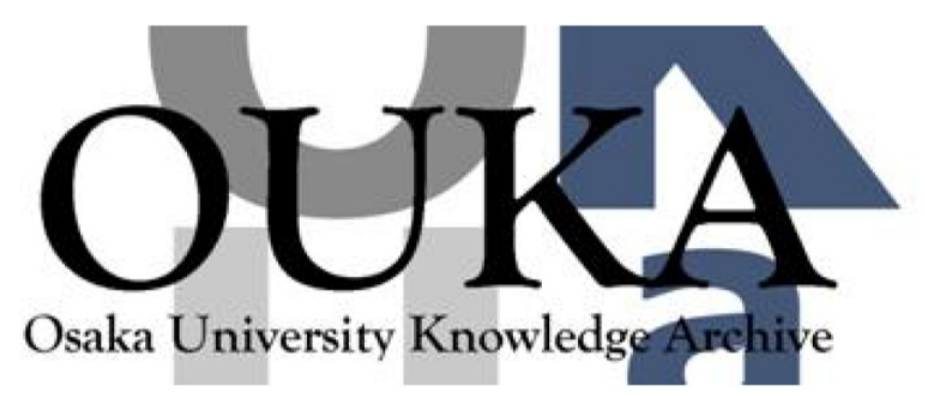

\begin{tabular}{|c|c|}
\hline Title & $\begin{array}{l}\text { EMAT pipe inspection technique using higher } \\
\text { mode torsional guided wave } T(0,2)\end{array}$ \\
\hline Author (s) & Nurmalia; Nakamura, N.; Ogi, H. et al. \\
\hline Citation & NDT \& E International. 87 p. 78-p. 84 \\
\hline Issue Date & $2017-04$ \\
\hline oaire:version & AM \\
\hline URL & https://hdl. handle. net/11094/84479 \\
\hline rights & $\begin{array}{l}\text { - } 2017 \text { Elsevier Ltd. This manuscript version is } \\
\text { made avai lable under the Creative Commons } \\
\text { Attribut ion-NonCommercial-NoDerivatives } 4.0 \\
\text { International License. }\end{array}$ \\
\hline Note & \\
\hline
\end{tabular}

Osaka University Knowledge Archive : OUKA

https://ir. Library. osaka-u. ac. jp/

0saka University 


\title{
EMAT pipe inspection technique using higher mode torsional guided wave $\mathbf{T}(0,2)$
}

\author{
Nurmalia* ${ }^{*}$ N. Nakamura*, H. Ogi, and M. Hirao
}

Graduate School of Engineering Science, Osaka University, Toyonaka, Osaka 560-8531, Japan

*Corresponding author: nobutomo@me.es.osaka-u.ac.jp

\begin{abstract}
We developed a pitch-catch system based on electromagnetic acoustic transducers (EMATs) for pipe inspection, which moves inside the pipe in the axial direction. The first higher mode, $\mathrm{T}(0,2)$, of the torsional guided wave is transmitted and detected, and variations of amplitude and phase are measured while moving the EMATs. Several aluminum pipes containing dish-shaped defects are inspected, and the amplitude and phase show enough detection sensitivity. It is found that the phase measurement has better potential as a tool for quantitative inspection. The applicability of the technique for steel pipe is also confirmed.
\end{abstract}

Keywords: EMAT, Torsional guided wave, Pipe inspection, Phase, Mode conversion

* Present address: Department of Engineering Physics, Bandung Institute of Technology, Bandung 40132, Indonesia 


\section{Introduction}

Pipes are critical components in infrastructure such as in petrochemical plants, oil and gas transportations, and public service installations. Pipelines often transport highly pressurized and flammable materials, where any failure could lead to a disaster. Integrity of the pipe must be carefully monitored and inspected regularly. Available methods for nondestructive pipe inspection can be classified into magnetic flux leakage [1, 2], eddy current [3, 4], x-ray radiography $[5,6]$, and ultrasonic $[7,8]$, in addition to visual inspection. The ultrasonic method using guided waves is superior over the others for its ability to inspect the whole-wall thickness and wide area in a short time.

The torsional wave offers a number of benefits compared to other guided waves because the component of particle displacement is only in the circumferential direction without involving the out-of-plane component [9]. This feature makes the torsional wave insensitive to liquid loading and insulation material. Furthermore, the dispersion behavior is simple and it is easy to analyze the received signals for practical inspection. These characteristics have drawn the attention of many researchers to utilize the torsional wave for pipe inspection [10-17].

An arrayed piezoelectric transducer $[15,18]$ and a magnetostrictive transducer $[8,19,20]$ were used in the previous experimental works on the torsional modes. In a piezoelectric transducer, a couplant is required and in a magnetostrictive transducer, a magnetostrictive patches must be bonded onto the outer surface of the pipes. In reality, the pipes are often buried in the ground and/or covered with insulators, and access to the pipes from the outside is not straightforward. In this study, we develop an arrayed periodic permanent magnet (PPM) electromagnetic acoustic transducers (EMATs) that can inspect the pipes from within the pipe. The PPM-EMAT is 
applied for inspection of defects fabricated on the outer surface of the pipes, and its ability for pipe inspection is demonstrated.

The fundamental mode, $\mathrm{T}(0,1)$, is usually focused upon because it is non-dispersive. In contrast, the present experimental study focuses on the higher mode, $\mathrm{T}(0,2)$. The idea of using $\mathrm{T}(0,2)$ mode is motivated by the phenomena observed in the previous studies by the authors $[16,17]$. When the $\mathrm{T}(0,2)$ mode encounters a thinning region in a pipe, the group velocity changes depending on the dispersive characteristic. We also observed that when the remaining thickness is smaller than a critical thickness (cut-off thickness), mode conversion from the $\mathrm{T}(0,2)$ mode to the $\mathrm{T}(0,1)$ mode occurs, which causes abrupt increment of the group velocity. The remaining thickness that is thinner than the cut-off thickness is then detectable from the changes in the group velocity. We also observed a total reflection when the wall thickness changes gradually around the thinning region [17]. These features should be applicable to the pipe inspection. In the presents study, an inspection is performed on several pipes containing dish-shaped defects, which resembles those induced by corrosion [21]. The ability of the mode-conversion based inspection using the developed PPM-EMAT is confirmed.

\section{Arrayed PPM-EMAT for Torsional Waves}

The arrayed PPM-EMAT designed for pipe inspection from the interior is illustrated in Fig. 1. The EMAT generates and detects the waves relying on the Lorentz force mechanism [22-24]. An eddy current in the axial direction is provided by a flat coil made from a wound copper wire of $0.2 \mathrm{~mm}$ in diameter. The coil is bent to follow the curvature of the pipe. One EMAT consists of a connection of four identical coils. The coil circuitry establishes an eddy current induced by the adjacent coils to be in the same axial direction. A static magnetic field in the thickness direction 
is provided by an arrayed PPM of Nd-Fe-B magnets, which are also designed to accommodate the curvature of the pipe. The arrayed PPM are arranged such that the cross-product of the eddy current and the static magnetic field results in the Lorentz force acting in the circumferential direction, as illustrated in the figures. This Lorentz force eventually induces an acoustic wave in the pipe. The PPM provides a periodically alternating magnetization in the axial direction, and this periodicity determines the wavelength, $\lambda$, of the generated torsional waves $(\lambda=5.22 \mathrm{~mm}$ in this study). The two EMATs, one for transmission and the other for detection, are separated by a fixed distance of $150 \mathrm{~mm}$ (center to center). They are placed on a suspension rod equipped with ball bearings at each end, enabling scanning measurement in the axial direction. The RF-bursts of 8 cycles are fed to the transmitting EMAT. The receiving EMAT also detects a specific signal of the same wavelength as the magnet periodicity. Furthermore, using the superheterodyne processing, it exclusively detects the signal having the same frequency component as the excitation RF-bursts. It is then possible to detect the intended torsional mode signal while excluding the other modes and noises. The typical equipment used for experiment can be found elsewhere $[16,17]$.
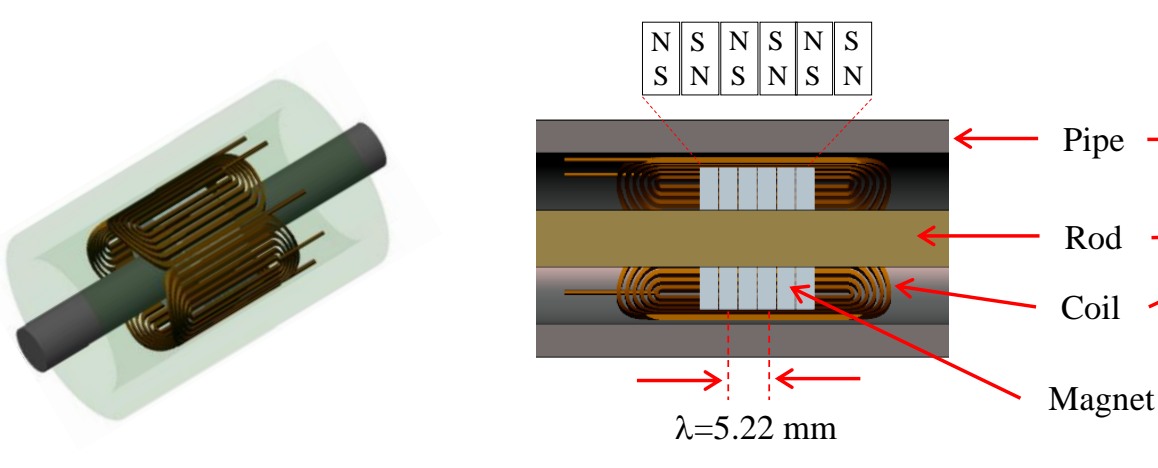

Cross-section

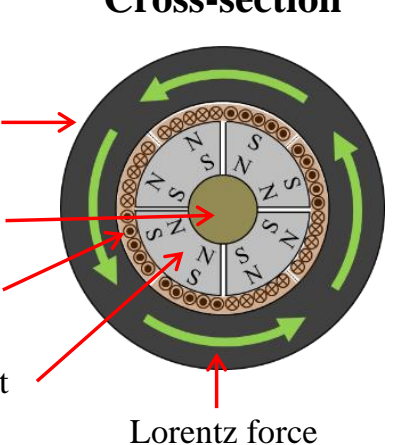

Figure 1 Configuration of the torsional-wave EMAT inside a pipe. Cross-sectional view normal to the axis shows the Lorentz force generation. The images are not to scale. 


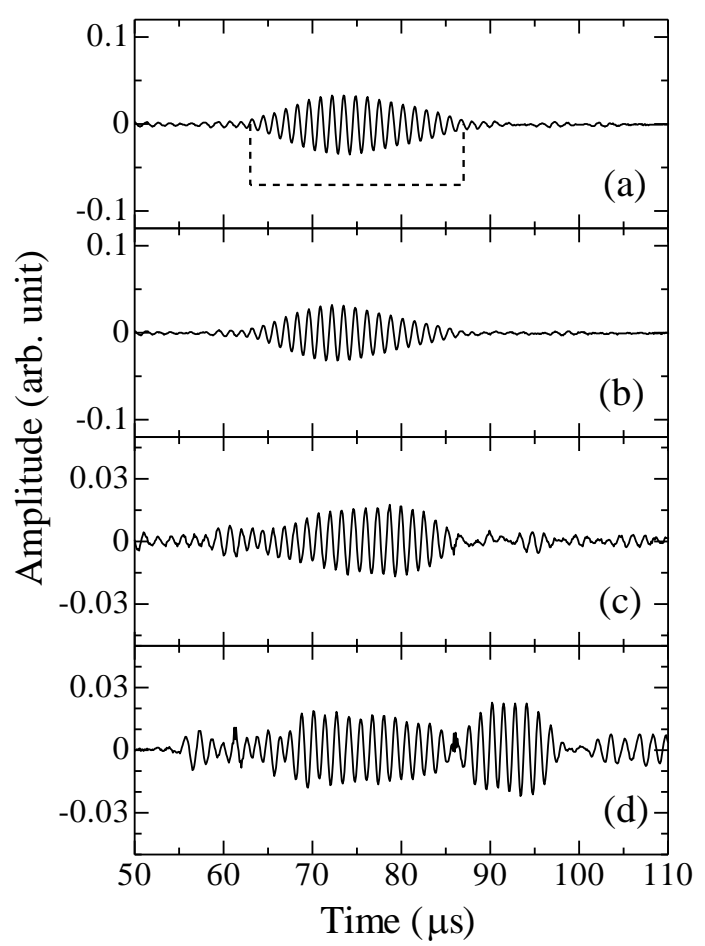

Figure 2 Typical signals in a defect-free (a) and defective (b) regions received by the EMAT, and those in a defect-free (c) and defective (d) regions received by a piezoelectric transducer in a pipe containing partial-circumferential defect. Minimum thickness and circumferential length of the defect is $2.0 \mathrm{~mm}$ and $61.8 \%$ of the full circumferential length, respectively. The EMAT detects only $\mathrm{T}(0,2)$ torsional modes. Dashed rectangle indicates the gate.

Figures 2(a) and 2(c) shows the typical signals, when a $T(0,2)$ mode is generated by the transmitting EMAT and detected by the receiving EMAT and by a needle-type piezoelectric transducer, respectively, in an original flawless pipe. Figures 2(b) and 2(d) compare signals when the $\mathrm{T}(0,2)$ mode is detected by the receiving EMAT and by a needle-type piezoelectric transducer in a pipe containing a partial-circumferential defect. Such a defect can be a source of other modes through mode conversions, and all the modes involved are received by the 
piezoelectric transducer (Fig.2 (d)). However, they are not detected by the EMAT (Fig. 2(b)), indicating high mode selectivity of the EMAT.

Figure 3 shows the group velocity dispersion curves of torsional modes for an aluminum pipe of $25 \mathrm{~mm}$ outer diameter ( $3 \mathrm{~mm}$ thick) and a steel pipe of $25.4 \mathrm{~mm}$ outer diameter ( $3.5 \mathrm{~mm}$ thick) used in the present study calculated with the following equation [25],

$$
\begin{aligned}
& {\left[\frac{1}{2}\left\{J_{0}(\kappa a)-J_{2}(\kappa a)\right\}-\frac{J_{1}(\kappa a)}{\kappa a}\right] \cdot\left[\frac{1}{2}\left\{Y_{0}(\kappa b)-Y_{2}(\kappa b)\right\}-\frac{Y_{1}(\kappa b)}{\kappa b}\right]-} \\
& {\left[\frac{1}{2}\left\{Y_{0}(\kappa a)-Y_{2}(\kappa a)\right\}-\frac{Y_{1}(\kappa a)}{\kappa a}\right] \cdot\left[\frac{1}{2}\left\{J_{0}(\kappa b)-J_{2}(\kappa b)\right\}-\frac{J_{1}(\kappa b)}{\kappa b}\right]=0 .}
\end{aligned}
$$

Here, $\kappa=\sqrt{\left(\omega^{2} / c_{s}^{2}\right)-\gamma^{2}}, \omega=2 \pi f, \gamma=2 \pi / \lambda$ with $f$ as the frequency, $c_{s}$ the shear wave velocity, $a$ and $b$ are inner and outer radii of the pipe, while $J_{i}$ and $Y_{i}$ are Bessel functions of the first and second kinds, respectively. For comparison, the dispersion curves of shear horizontal

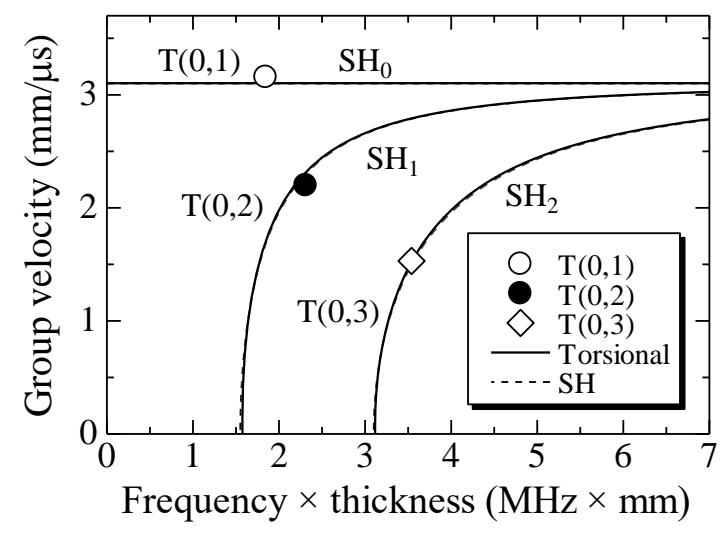

(a)

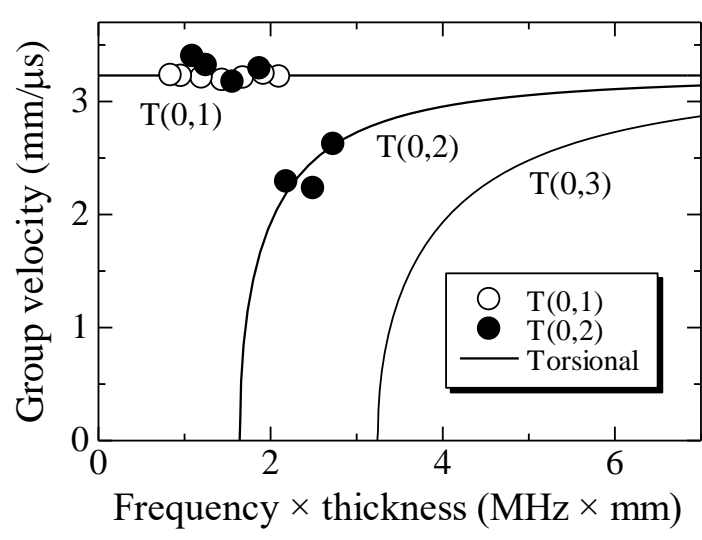

(b)

Figure 3 Group velocities measured by the EMATs and theoretical dispersion curves for aluminum (a) and steel pipes (b). Individual points are the measurements. Solid and dashed lines in (a) are the dispersion curves calculated for torsional and SH modes, respectively. 
(SH) waves, propagating in an aluminum plate of the same thickness as the pipe is superimposed. They are nearly the same, indicating that the dispersion relation for a plate, simpler than that for a pipe, is well applicable to analyze the measured group velocities when the wavelength is sufficiently shorter than the pipe diameter.

The $\mathrm{T}(0,2)$ mode is of focus in the present study, but the developed EMATs are actually capable of generating and detecting some other torsional modes: $\mathrm{T}(0,1)$ and $\mathrm{T}(0,3)$ modes in an aluminum pipe and $\mathrm{T}(0,1)$ mode in a steel pipe. Driving frequencies of $\mathrm{T}(0,1), \mathrm{T}(0,2)$, and $\mathrm{T}(0,3)$ modes in an aluminum pipe are $0.615,0.77$, and $1.18 \mathrm{MHz}$, respectively. Those of $\mathrm{T}(0,1)$ and $\mathrm{T}(0,2)$ modes for a steel pipe are 0.6 and $0.78 \mathrm{MHz}$, respectively. Their measured group velocities are also plotted in Fig. 3. A good agreement between the measured group velocities and the theoretical dispersion curves provides confidence that the group velocities of torsional modes are measured using the present setup. For steel pipes, specimens containing fullcircumferential defects with step edge were prepared and group velocities at the defects were measured for each mode, which are also plotted in the figure. The data experimentally confirms the thickness dependence of group velocity. A jump in the group velocity is observed, indicating the mode conversion to $\mathrm{T}(0,1)$ mode. However, it occurs at a larger thickness than the theoretical cut-off thickness of $2.1 \mathrm{~mm}$. The experimental cut-off thickness exists between $2.4 \mathrm{~mm}$ and 2.8 $\mathrm{mm}$. The reason for this discrepancy is unknown at the moment. The similar data for aluminum pipes can be found in the previous work [16].

Scanning inspection is realized by pulling the suspension rod from the outside by a controllable motor via a thin cord. In the following analysis, the intended signal, surrounded by the dashed line in Fig. 2(a), is gated, and its phase and amplitude are measured. The motor provides a very slow linear movement of the EMATs in the axial direction, while the phase and amplitude are 
continuously measured. Measurement of a set of amplitude and phase typically takes two seconds when five signals are averaged.

\section{Results and Discussion}

\subsection{Full Circumference Defect}

Aluminum pipes with dimensions of $25 \mathrm{~mm}$ outer diameter and $3 \mathrm{~mm}$ thick wall are used as specimens. Different sizes of dish-shaped defects, around the entire circumference of the pipe, were fabricated in the middle of each specimen. All of the defects were introduced by using an $80 \mathrm{~mm}$ diameter end mill. The rotating end mill is placed normal to the axis of the pipe at its center and lowered until the desired remaining wall thickness is achieved. The process is repeated every 15 degrees by rotating the pipes until the entire circumference has been uniformly cut. Different remaining wall thickness results in a different axial length of the defect, while maintaining the same curvature radius. Minimum wall thickness, $h_{m}$, axial length, $L$, and relative axial length to the wavelength, $L / \lambda$, of all defects are shown in Table 1 . Schematic image of a defect is shown in Fig. 4.

Table 1 Dimensions of the full circumferential defects

\begin{tabular}{|l|l|l|l|}
\hline Specimen & $\begin{array}{l}\text { Minimum wall } \\
\text { thickness, } h_{m},(\mathrm{~mm})\end{array}$ & $L(\mathrm{~mm})$ & $L / \lambda$ \\
\hline 1 & 2.5 & 12.6 & 2.4 \\
\hline 2 & 2.0 & 17.4 & 3.3 \\
\hline 3 & 1.9 & 19.0 & 3.6 \\
\hline 4 & 1.5 & 21.6 & 4.1 \\
\hline 5 & 0.6 & 27.6 & 5.3 \\
\hline
\end{tabular}




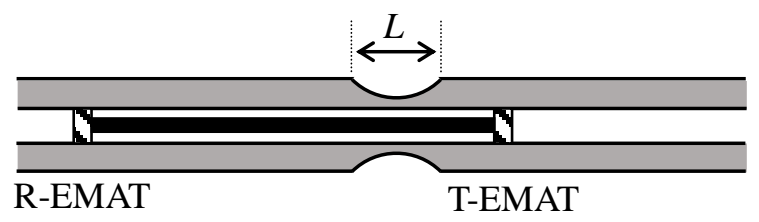

(a)

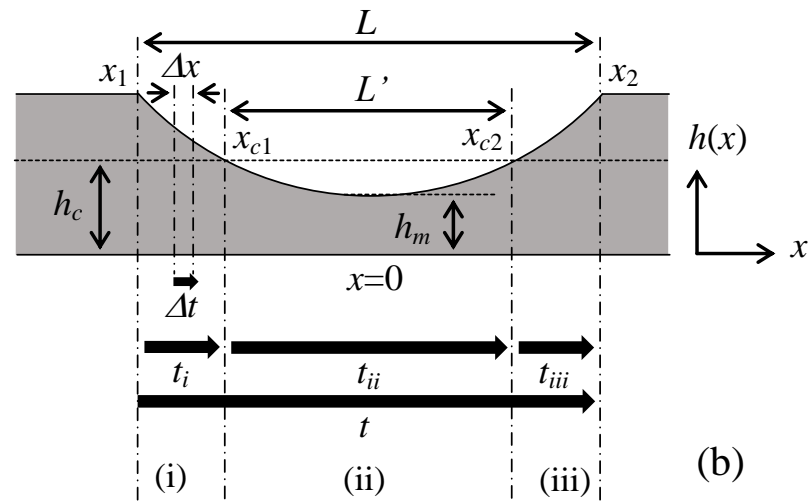

Figure 4 Defect location between the two EMATs (a), and a 2D model of the defect (b). The defect dimension is exaggerated for clarity.

Figures 5 shows the typical amplitude and relative phase profiles of some specimens. The amplitude is normalized by that at a flawless region. The center of defect is located at $x=0$. It is obvious that the presence of a defect alters both the amplitude and phase profiles. The length at which the alteration occurs is close to the distance between the two EMATs. The amount of amplitude and phase changes in all specimens are shown in Fig. 6. 

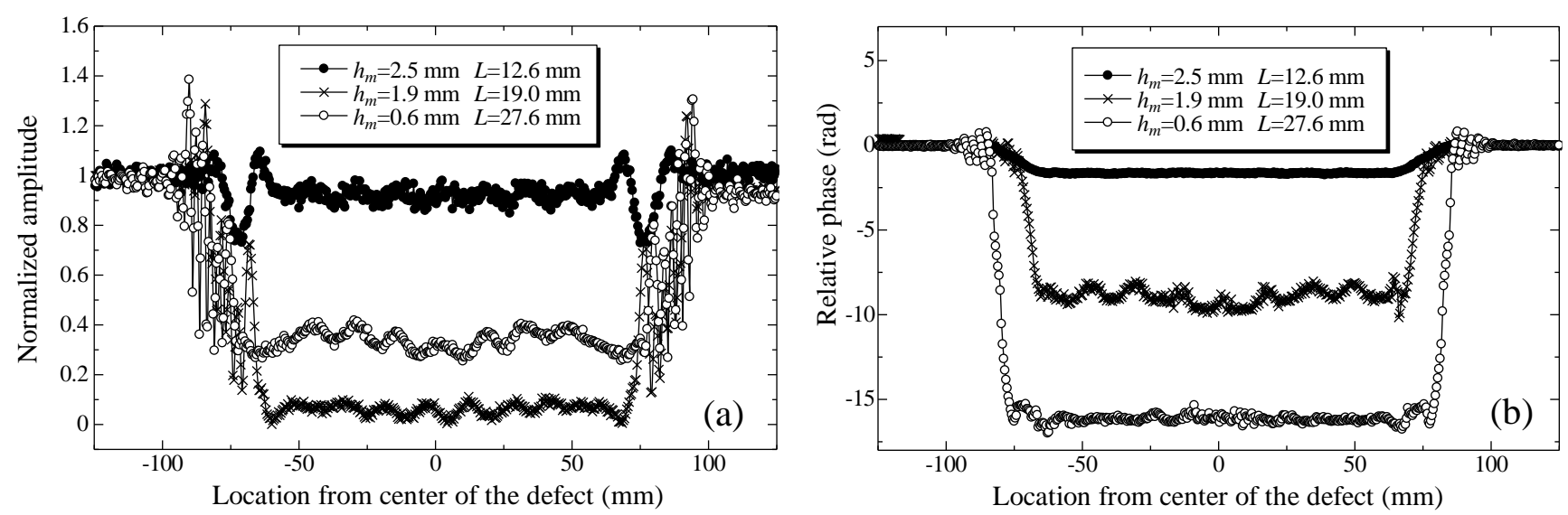

Figure 5 Amplitude (a) and phase (b) profiles of some specimens showing the influence of a defect in the middle.

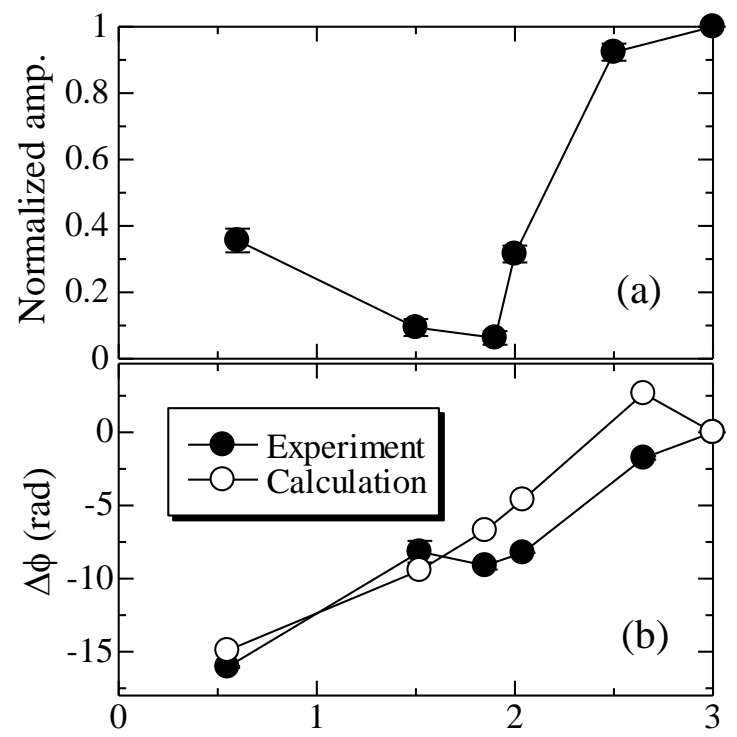

Minimum remaining thickness $h_{m}(\mathrm{~mm})$

Figure 6 Amplitude (a) and phase (b) changes caused by the defects with different remaining thickness. The phase changes show a simpler trend when compared to the amplitude. Open circles in (b) are calculated from the model. 
The minimum wall thickness at the defect of specimens $2-5$ is smaller than the cut-off thickness, $2.35 \mathrm{~mm}$, of $\mathrm{T}(0,2)$ mode [16]. In the previous study [17], it was observed that when the remaining thickness is smaller than the cut-off thickness, as the slope of the defect's edge becomes smaller, the reflectivity of the $\mathrm{T}(0,2)$ mode increases. In Fig. 6(a), amplitude of the transmitted waves becomes smaller as the remaining thickness decreases, indicating the increase in the reflectivity of the $\mathrm{T}(0,2)$ mode. However, as the remaining thickness decreases, the slope at the edges of a defect becomes larger, resulting in a decrease in the reflectivity. This is a possible reason of the increase in the amplitude of the longest specimen (specimen 5). For specimen 1 with the smallest defect, remaining thickness at the defect is larger than the cut-off thickness and the mode can exist in both the defect-free and the defective sections. The reflection hardly occurs at the defect, resulting in the less decrement of the amplitude. However, the notable depressions at the edges of the defect are observed (Fig.5(a)). These observations occur when one of the EMATs is located right below the defect, suggesting that the pipe wall-thickness significantly affects the generation and detection efficiency of the EMATs. Thus, the amplitude of $\mathrm{T}(0,2)$ mode can be used as a parameter for defect detection. But, it is not sufficient enough for sizing purpose of a fully circumferential dish-shaped defect.

Relationship between the phase changes and the remaining thickness at the defect is more straightforward, as demonstrated in Fig. 6(b); the phase shows a decreasing trend as the minimum remaining thickness decreases. For specimens $2-5$ with remaining thicknesses smaller than the cut-off thickness, the mode conversion to $\mathrm{T}(0,1)$ mode with a higher velocity at a defect can explain the decrease. For the specimen 1, however, according to the dispersion curve of Fig. 3(a), the group velocity of $\mathrm{T}(0,2)$ mode at the defect should have been smaller than that at 
the flawless region, resulting in an increase in the phase. However, the result shows an opposite behavior; the phase slightly decreases at the defect. The reason remains unknown.

A decreasing trend in phase is observed following the decrease in the minimum thickness, suggesting that the phase decrease is a better parameter for defect sizing when compared to the amplitude. For this purpose, a quantitative analysis of the phase change is considered. The decrease in the phase scanning profile in Fig. 5(b) appears when the defect is located between the two EMATs. In this case, the detected waves have propagated in both the defect-free and the defect sections as illustrated in Fig. 4(a). The decrease in the phase, $\Delta \phi=2 \pi \Delta t$, is then induced by the difference of propagating time, $\Delta t=t-t_{0}$, required to travel a distance $L$ with the defect, $t$, and without the defect, $t_{0}$. The defect itself can be divided as three regions: two sloping regions (i) and (iii) where thickness is larger than the cut-off thickness, $h_{c}$, and region (ii) where thickness is smaller than $h_{c}$, as illustrated in Fig. 4(b). The total traveling time over $L$ is then the sum of traveling times in all regions,

$t=t_{i}+t_{i i}+t_{i i i}$

In region (ii) of length $L^{\prime}$, mode conversion to the fundamental $\mathrm{T}(0,1)$ mode occurs and the traveling time in the region is expressed as:

$t_{i i}=\frac{x_{c 2}-x_{c 1}}{c_{g_{[\mathrm{T}(0,1)]}}}=\frac{L^{\prime}}{c_{s}}$.

Here, $c_{g[\mathrm{~T}(0,1)]}$ is the group velocity of the $\mathrm{T}(0,1)$ mode. As described in section 2 , the $\mathrm{T}(0,2)$ mode propagating in the specimens can be treated as the first higher shear-horizontal mode, $\mathrm{SH}_{1}$, propagating in a plate of the same thickness. For simplicity of the calculation, we use the 
dispersion relationship for a plate in the following analysis. Then, the group velocity of the $\mathrm{SH}_{1}$ mode is [26]:

$$
c_{g_{\left[\mathrm{SH}_{1}\right]}}=c_{s} \sqrt{1-\frac{c_{s}}{2 f h(x)}} .
$$

In the present measurements, the frequency $f$ is fixed and thickness $h(x)$ is the only variable contributing to the group velocity, which follows the equation of a circle:

$$
h(x)=\sqrt{40^{2}-x^{2}}-\left(40+h_{m}\right)
$$

By referring to Fig. 4(b), $t_{i}$ and $t_{i i i}$ can be determined as:

$$
t_{i}=t_{i i i}=\int_{x_{1}}^{x_{c 1}} \Delta t_{x 1}=\int_{x_{1}}^{x_{c 1}} \frac{d x}{c_{g_{\left[\mathrm{SH}_{1}\right]}}}=\int_{x_{1}}^{x_{c 1}} \frac{d x}{c_{s} \sqrt{1-\frac{c_{s}}{2 f h(x)}}} .
$$

In the case of specimen 1, the remaining thickness at defect is larger than the cut-off thickness. The traveling time, $t$, is then determined by Eq. (6) with the integration range from $x_{1}$ to $x_{2}$.

The calculated $\Delta \phi$ are plotted in Fig. 6(b) together with those obtained from experiment. For the defects with a minimum thickness $h_{m}$ smaller than $2.5 \mathrm{~mm}$ (or an axial length $L$ longer $12.6 \mathrm{~mm}$ ), both phases basically show a reduction. A linear relationship is observed between the phase decrease and the axial length $L^{\prime}$, over which the converted T( 0,1$)$ mode travels, as shown in Fig. 7. For the shortest defect, increment of the phase appears in the calculation, but decrement was observed in the experiment (Fig. 6(b)). Due to the limitation in the machining, the defects are actually not smooth; there is a variation of thickness along the circumference. This might contribute to the gap between the calculation and experiment. Nevertheless, these results indicate 
that the change in the phase can be applied to the quantitative evaluation of the defect's length for full circumferential defects, where the remaining thickness is smaller than the cut-off thickness. When defect shape is complicated, it will be difficult to determine the defect's length accurately. Then, we can use results of amplitude measurement. From the amplitude depressions at the edges of defect, defect's total length can be estimated. By calculating traveling time assuming different shapes of defects and by comparing it with experimental results, defect's length where the remaining thickness is smaller than the cut-off thickness will be estimated.

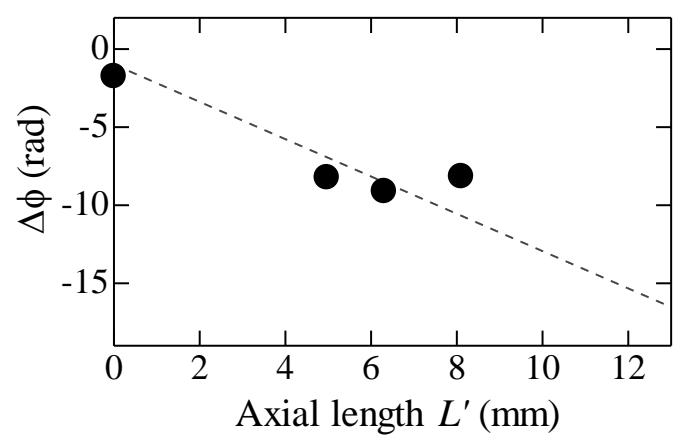

Figure 7 Relationship between the phase decrease and the axial length where thickness is smaller than the cut-off thickness, $L^{\prime}$. Dashed line shows the linear fitting.

\subsection{Partial Circumference Defect}

The scanning procedure is applied to the partially circumferential defects. Aluminum pipes categorized in three sets are prepared for this experiment. The dimensions of the pipes are also $25 \mathrm{~mm}$ in outer diameter and $3 \mathrm{~mm}$ thickness. The defects are made with the same procedures as for the full circumference defects, but the numbers of pipe rotations are different for each specimen. The sets of specimens consist of several pipes containing defects of the same depth but with varying overall lengths in circumferential direction. Table 2 shows the defect dimensions of all specimen where $L_{c}$ is the circumference of the defect and $L_{c 0}$ is that of the 
original pipe $(78.5 \mathrm{~mm})$. The longest circumferential of each defect, which is located at midpoint, is used to determine the $L_{c}$.

Table 2 Dimensions of partial defects

\begin{tabular}{|l|l|l|l|}
\hline & Spec. No. & $h_{m}(\mathrm{~mm})$ & $L_{c} / L_{c 0}(\%)$ \\
\hline \multirow{5}{*}{ Set-1 } & $1 \mathrm{a}$ & 2.5 & 30 \\
\cline { 2 - 4 } & $1 \mathrm{~b}$ & 2.5 & 58 \\
\cline { 2 - 4 } & $1 \mathrm{c}$ & 2.5 & 83.4 \\
\cline { 2 - 4 } & $1 \mathrm{~d}$ & 2.5 & 100 \\
\hline \multirow{5}{*}{ Set-2 } & $2 \mathrm{a}$ & 2.0 & 36.3 \\
\cline { 2 - 4 } & $2 \mathrm{~b}$ & 2.0 & 61.8 \\
\cline { 2 - 4 } & $2 \mathrm{c}$ & 2.0 & 87.3 \\
\cline { 2 - 4 } & $2 \mathrm{~d}$ & 2.0 & 100 \\
\hline \multirow{5}{*}{ Set-3 } & $3 \mathrm{a}$ & 1.9 & 37.6 \\
\cline { 2 - 4 } & $3 \mathrm{~b}$ & 1.9 & 63 \\
\cline { 2 - 4 } & $3 \mathrm{c}$ & 1.9 & 88.5 \\
\cline { 2 - 4 } & $3 \mathrm{~d}$ & 1.9 & 100 \\
\hline
\end{tabular}

The amplitude and phase changes in all specimens are shown in Figs. 8(a) and (b), respectively. Horizontal axis is the percentage of defective circumference. Relationship between the amount of amplitude change and the circumferential length of the defect is complicated, especially for Set-1. The defects of specimen Sets- 2 and 3 contain a region where the wall thickness is smaller than the cut-off thickness of $2.3 \mathrm{~mm}$. The amplitude at the defect of these specimens shows a decreasing trend as the defect's circumferential length increases, particularly in the last three specimens (from the half to full-circumference defect). In the pipes containing partial circumference defects, an interference occurs between the waves propagating at the defective 
and defect-free parts in the same axial plane of the pipe. This interference induces a complicated relationship between the amplitude changes and the defective circumference lengths. Figure 8(a) indicates that amplitude change is not suitable for quantitative evaluation of a partial circumference defect, particularly for a shallower defect.

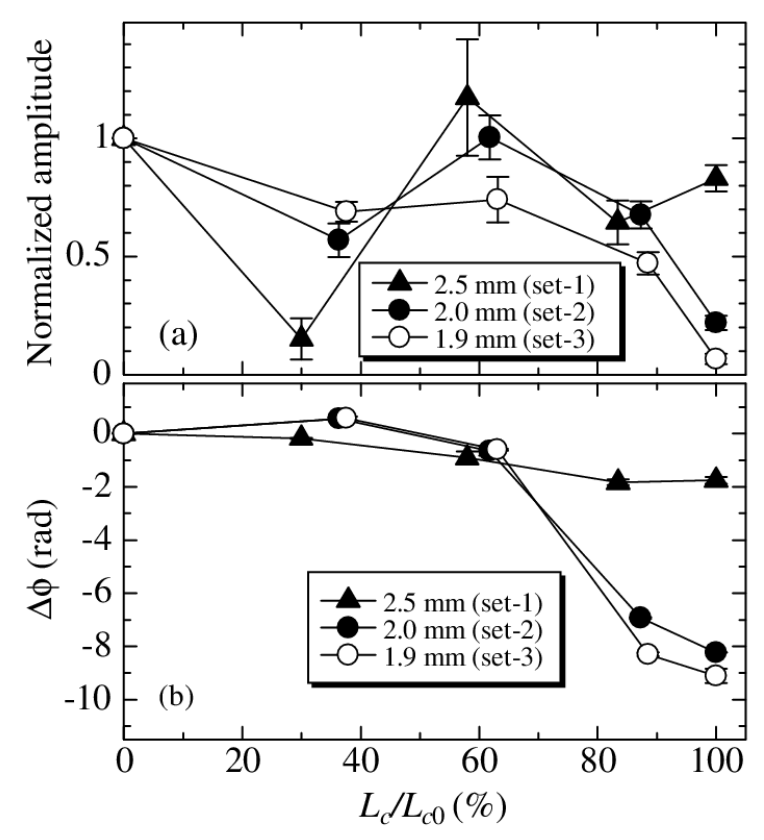

Figure 8 Amplitude (a) and phase (b) changes in all specimens containing partial defects. The phase change shows a simpler relationship when compared to the amplitude.

The phase changes are relatively small in specimen Set-1 with shallower defects, as can be seen in Fig. 8(b). In the other two sets, phase slightly increases at the defects with the shortest circumferential lengths (specimens $2 \mathrm{a}$ and $3 \mathrm{a})$. In these specimens, the generated $\mathrm{T}(0,2)$ mode takes a detour around the defect, causing a slightly increased traveling distance. The phase decreases significantly in the last two specimens of each set with longer defective circumferences. This phase drop is attributed to a conversion to the $\mathrm{T}(0,1)$ mode with a higher group velocity, as explained in the sub section 3.1 . The contribution of converted $T(0,1)$ mode 
increases as the defective circumference becomes longer. The phase at defects slightly decreases for specimen $2 \mathrm{~b}$ and $3 \mathrm{~b}$. This could be a result of the competing effects between a detour around the defect and the mode conversion.

To date, steel pipes are most often used for various applications. It is then necessary to verify the applicability of the proposed technique for inspection of steel pipes. Generating torsional waves in steel material is not as straightforward as in aluminum. The reasons are a larger mass density and a higher electric resistance than aluminum, lowering the excitation efficiency of the torsional modes. An additional technical problem arises when applied in a steel pipe: the pipe wall attracts the permanent magnets of the EMATs.

A scanning inspection is performed on a steel pipe with an outside diameter of $25.4 \mathrm{~mm}$ and 3.5 $\mathrm{mm}$ thick wall. A defect is introduced on it manually using a half-round file. The defect is 10 $\mathrm{mm}$ in axial and $25 \mathrm{~mm}$ in circumferential lengths. The depth of defect is in the range of $0.8-$ $0.9 \mathrm{~mm}$; it is not exactly uniform since the defect is made by hand. According to the measured group velocities in Fig. 3(b), the mode conversion may occur at defect because the minimum thickness at the current defect is in the range of $2.6-2.7 \mathrm{~mm}$.

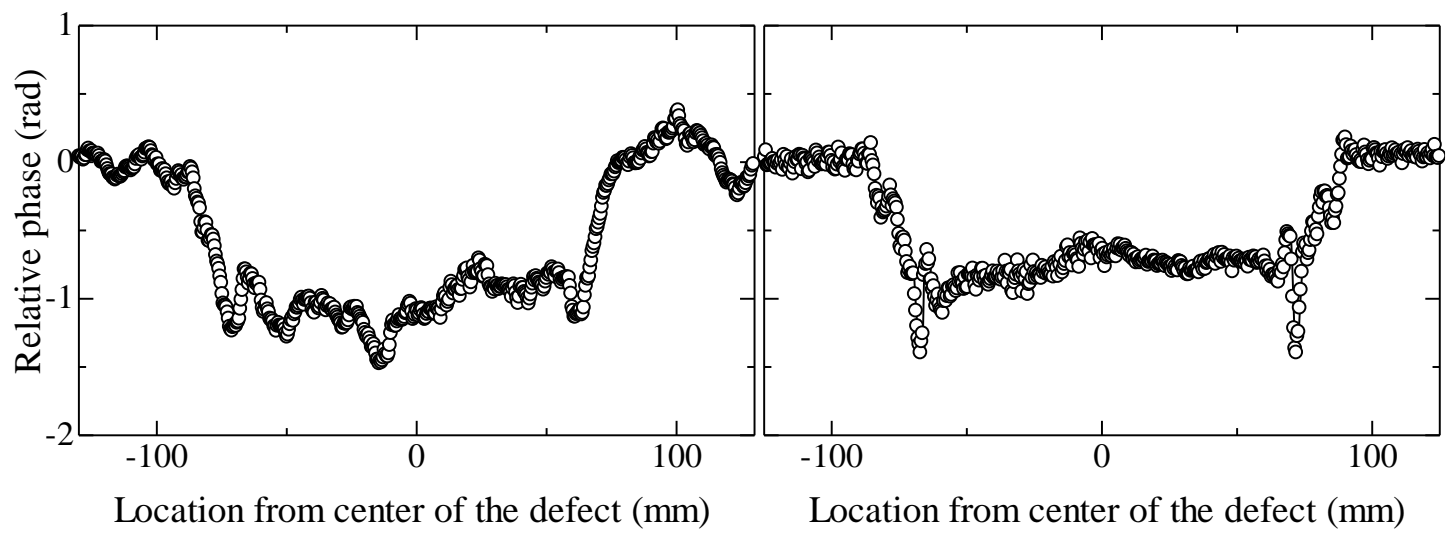

Figure 9 Phase profiles of steel (a) and aluminum (b) pipes containing partial defects. 
The obtained relative phase profile is shown in Fig. 9(a). The phase reduces about 1 radian by the presence of the defect. Since the defect covers less than half of the circumference, the effect of a detour around the defect likely exists in addition to the mode conversion effect. It is observed nonetheless that the effect of conversion to the $\mathrm{T}(0,1)$ mode at the defect dominates and results in a reduced traveling time. The observed fluctuation at the defective region in steel pipe is fairly comparable to those in aluminum pipes. The phase profile of specimen $2 \mathrm{~b}$ is shown in Fig. 9(b) as an example. It is then confirmed that the scanning technique is also applicable for the inspection of a steel pipe.

\section{Conclusion}

This study utilized a higher torsional mode, $\mathrm{T}(0,2)$, for pipe inspection. A pair of EMATs was used to generate and detect the mode, which was designed for a scanning inspection performed from within a pipe. The technique was used to inspect several pipes containing dish-shaped defects of various dimensions. The changes in the amplitude and phase profiles caused by the defects were investigated to find their correlation with the defect size. Both the amplitude and phase were altered by a defect of any size, meaning that the technique was basically effective for the purpose of flaw detection. However, the phase change in general showed more potential for quantitative evaluation. For the full circumference defect, the mode conversion to the fundamental mode could explain the observed phase changed. For the partial circumference defect, two phenomena with the opposite effect were considered as the origins of a phase change: (i) the mode conversion at the site of a defect to the $\mathrm{T}(0,1)$ mode with a higher group velocity, which induced a reduction in phase, (ii) the detour around the defect that resulted in a longer 
traveling path and led to an increase in phase. It was observed that the contribution of the mode conversion effect increased as the defective circumference became longer. The scanning measurements were also performed in a steel pipe containing a similar defect and yielded results that confirmed the applicability of the proposed technique for inspecting steel material.

Table 3 summarizes the results of the amplitude and phase measurements of the $\mathrm{T}(0,2)$ mode with the current technique for defect detection and sizing purposes. The symbols $\mathrm{O}, \Delta$, and $\times$ mean good, unsatisfactory, and poor, respectively. In the present study, we inspected defects with simple and smooth shapes. However, shape of actual defects should be more complicated, and criteria in Table 3 may not be necessarily applicable to them; the criteria could be improved after further investigation on various shapes of defects to use the proposed method in the field inspection. In addition, it is rare that one would know, a priori, if the defect is completely circumferential or not. However, the proposed method can estimate the shape. Detour around a partial defect and slight wall thinning generally increase the traveling time between EMATs, but the mode conversion from $\mathrm{T}(0,2)$ to $\mathrm{T}(0,1)$ mode decreases the traveling time. Considering that the mode conversion occurs when the remaining wall thickness at the defect is less than the cutoff thickness and the circumferential size is larger than $80 \%$ of the circumferential full length, observation of the relative phase decrement indicates the presence of a defect that is larger than the above scales, even when the defect shape is complicated and quantitative measurement is difficult. 
Table 3 Summary of results.

\begin{tabular}{|l|l|l|l|l|l|l|}
\hline \multirow{2}{*}{} & \multicolumn{2}{|l|}{ Full defect } & \multicolumn{2}{l|}{ Partial defect $\left(h_{m}>h_{c}\right)$} & \multicolumn{2}{l|}{ Partial defect $\left(h_{m}<h_{c}\right)$} \\
\cline { 2 - 7 } & Detection & Sizing & Detection & Sizing & Detection & Sizing \\
\hline Amplitude & $\mathrm{O}$ & $\Delta$ & $\mathrm{O}$ & $\times$ & $\mathrm{O}$ & $\times$ \\
\hline Phase & $\mathrm{O}$ & $\mathrm{O}$ & $\mathrm{O}$ & $\Delta$ & $\mathrm{O}$ & $\mathrm{O}$ \\
\hline
\end{tabular}




\section{References}

1. Saha S, Mukhopadhyay S, Mahapatra U, Bhattacharya S, Srivastava GP. Empirical structure for characterizing metal loss defects from radial magnetic flux leakage signal. NDT \& E Int 2010;43:507- 512.

2. Liu B, Cao Y, Zhang H, Lin YR, Sun WR, Xu B. Weak magnetic flux leakage: A possible method for studying pipeline defects located either inside or outside the structures. NDT \& E Int 2015;74:81-86.

3. Nestleroth JB, Davis RJ. Application of eddy currents induced by permanent magnets for pipeline inspection. NDT \& E Int 2007;40:77 - 84.

4. Xu Z, Wu X, Li J, Kang Y. Assessment of wall thinning in insulated ferromagnetic pipes using the time-to-peak of differential pulsed eddy-current testing signals. NDT \& E Int 2012;51: $24-29$.

5. Kersting T, Schönartz N, Oesterlein L, Liessem S. High end inspection by filmless radiography on LSAW large diameter pipes. NDT \& E Int 2010;43:206 - 209.

6. Movafeghi A, Yahaghi E, Mohammadzadeh N. Defect detection improvement of digitized radiographs by principal component analysis with local pixel grouping. J Nondestruct Eval $2015 ; 34: 17$.

7. Hirao M, Ogi H. An SH-wave EMAT technique for gas pipeline inspection. NDT \& E Int $1999 ; 32: 127-132$.

8. Kim YY, Kwon YE. Review of magnetostrictive patch transducers and applications in ultrasonic nondestructive testing of waveguides. Ultrasonics 2015;62:3 - 19 .

9. Achenbach JD. Wave propagation in elastic solids. $8^{\text {th }}$ ed. Amsterdam: Elsevier; 1999.

10. Demma A, Cawley P, Lowe M. The reflection of the fundamental torsional mode from 
cracks and notches in pipes. J Acoust Soc Am 2003;114: 611 - 625.

11. Ratassepp M, Fletcher S, Lowe MJS. Scattering of the fundamental torsional mode at an axial crack in a pipe. J Acoust Soc Am 2010;127:730 - 740.

12. Carandente R, Cawley P. The effect of complex defect profiles on the reflection of the fundamental torsional modes in pipes. NDT \& E Int 2012;46:41 - 47.

13. Kirby R, Zlatev Z, Mudge P. On the scattering of torsional elastic waves from axisymmetric defects in coated pipes. J Sound Vibration 2012;331:3989 - 4004.

14. Løvstad A, Cawley P. The reflection of the fundamental torsional mode from pit clusters in pipes. NDT \& E Int 2012;46:83 - 93.

15. Nishino H, Tanaka T, Katashima S, Yoshida K. Experimental investigation of mode conversion of the T(0,1) mode guided wave propagating in an elbow pipe. Jpn J Appl Phys 2011;50:046601.

16. Nurmalia, Nakamura N, Ogi H, Hirao M. Mode conversion of torsional waves generated by electromagnetic acoustic transducer. AIP Conf Proc 2013;1511:909 - 915.

17. Nurmalia, Nakamura N, Ogi H, Hirao M. Mode conversion and total reflection of torsional waves for pipe inspection. Jpn J Appl Phys 2013;52:07HC14.

18. Liu Z, He C, Wu B, Wang X, Yang S. Circumferential and longitudinal defect detection using $\mathrm{T}(0,1)$ mode excited by thickness shear mode piezoelectric elements. Ultrasonics 2006;44:e1135 - e1138.

19. Kim YY, Park CI, Cho SH, Han SW. Torsional wave experiments with a new magnetostrictive transducer configuration. J Acoust Soc Am 2005;117:3459 - 3468.

20. Kim YG, Moon HS, Park KJ, Lee JK. Generating and detecting torsional guided waves using magnetostrictive sensors of crossed coils. NDT \& E Int 2011;44:145 - 151. 
21. Majid ZA, Mohsin R, Yaacob Z and Hassan Z. Failure analysis of natural gas pipes. Eng Fail Anal 2012;17:818-837.

22. Thompson RB. Physical principles of measurements with EMAT transducers. In: R.N. Thurston, A.D. Pierce, editors. Physical Acoustics, 19. San Diego: Academic Press; 1990. p. $157-200$.

23. Hirao M, Ogi H. Electromagnetic acoustic transducer: Noncontacting ultrasonic measurements using EMATs: Springer; 2017.

24. Vasile CF, Thompson RB. Excitation of horizontally polarized shear elastic waves by electromagnetic transducers with periodic permanent magnets. J Appl Phys 1979;50(4):2583 -2588 .

25. Gazis DC. Three-dimensional investigation of the propagation of waves in hollow circular cylinders. I. Analytical foundation. J Acoust Soc Am 1959;31:568 - 573.

26. Nurmalia, Nakamura N, Ogi H, Hirao M, Nakahata K. Mode conversion behavior of SH guided wave in a tapered plate. NDT \& E Int 2012;45:156 - 161. 


\section{Figure Captions}

Figure 1 Configuration of the torsional-wave EMAT inside a pipe. Cross-sectional view normal to the axis shows the Lorentz force generation. The images are not to scale.

Figure 2 Typical signals in a defect-free (a) and defective (b) regions received by the EMAT, and those in a defect-free (c) and defective (d) regions received by a piezoelectric transducer in a pipe containing partial-circumferential defect. Minimum thickness and circumferential length of the defect is $2.0 \mathrm{~mm}$ and $61.8 \%$ of the full circumferential length, respectively. The EMAT detects only $\mathrm{T}(0,2)$ torsional modes. Dashed rectangle indicates the gate.

Figure 3 Group velocities measured by the EMATs and theoretical dispersion curves for aluminum (a) and steel pipes (b). Individual points are the measurements. Solid and dashed lines in (a) are the dispersion curves calculated for torsional and SH modes, respectively.

Figure 4 Defect location between the two EMATs (a), and a 2D model of the defect (b).

The defect dimension is exaggerated for clarity.

Figure 5 Amplitude (a) and phase (b) profiles of some specimens showing the influence of a defect in the middle.

Figure 6 Amplitude (a) and phase (b) changes caused by the defects with different remaining thickness. The phase changes show a simpler trend when compared to the amplitude. Open circles in (b) are calculated from the model.

Figure 7 Relationship between the phase decrease and the axial length where thickness is smaller than the cut-off thickness, $L^{\prime}$. Dashed line shows the linear fitting. 
Figure 8 Amplitude (a) and phase (b) changes in all specimens containing partial defects. The phase change shows a simpler relationship when compared to the amplitude.

Figure 9 Phase profiles of steel (a) and aluminum (b) pipes containing partial defects. 